\title{
Statistical Research on Pulsars with the Gigahertz-Peaked Spectra
}

\author{
Hongbing $\mathrm{CAI}^{\mathrm{a}, \mathrm{b}, 1}$, Jingsong $\mathrm{XU}^{\mathrm{a}, \mathrm{b}}$, and Beibei $\mathrm{WANG}^{\mathrm{a}, \mathrm{b}}$ \\ a Jiangsu Normal University, Xuzhou 221116, China \\ ${ }^{\mathrm{b}}$ Xuzhou Huitian Information Technology Co., Ltd, Xuzhou 221010, China
}

\begin{abstract}
The pulsar's radio spectra in the high frequency shows the power law form in general, but people has also found 23 pulsars with the turn-over spectra peaking near $\mathrm{GHz}$, i.e., the Gigahertz-peaked spectrum (GPS) pulsars. We launch the statistical research on these GPS pulsars, and find the peak frequency of $73.9 \%$ GPS pulsars in the sample is between $0.5 \mathrm{GHz}$ and $1.0 \mathrm{GHz}$. The magnetic star has the higher peak frequency relative to other GPS pulsars, whose average peak frequency is $3.35 \mathrm{GHz}$. The average spectral index of GPS pulsars is -1.33 which is higher than ones of ordinary pulsars. This phenomenon may be caused by the interstellar medium absorption for the pulsar's radiation in the low frequency band. The statistical average of the dispersion measure of GPS pulsars is $348 \mathrm{pc} \mathrm{cm}^{-3}$, which is higher than the ones of ordinary pulsars. We also find the strong positive correlation between the period and dispersion measure of GPS pulsars, which imply that the interstellar medium may cause the pulsar's rotation slow down.
\end{abstract}

Keywords. Pulsar, radio spectra.

\section{Introduction}

Since the discovery of pulsar in 1967, pulsar is always the research highlight in the astronomical and physical fields due to its extreme physical properties such as strong magnetic field, strong gravitation, super stable rotation period, but also pulsar is the only celestial body which can be directly observed. The radio spectra of pulsar can help us probe into its radiating mechanism, which is conducive to the research on the formation and evolution of pulsar. The previous research shows the radio spectrum of pulsars may be represented using the power law form, the spectral index is about -1.8 [1]. But Sieber found as early as 1973 that some pulsars' spectrum have the turn-over phenomenon, and Sieber used the synchrotron self-absorption and free-free absorption models to fit the turn-over spectrum. This paper could not make clear which model is better due to the limit of observing data. Koribalski et al. studied 8 pulsars by using the 64-metre Parkes radio telescope and found the GPS pulsar PSR B1054-62 is sheltered by hydrogen ionization zone or locates in the hydrogen ionization zone [2]. This discovery provided the observing evidence that the turn-over spectra of pulsar may be caused by the free-free absorption from electrons.

\footnotetext{
${ }^{1}$ Corresponding Author, Hongbing CAI, Jiangsu Normal University, Xuzhou 221116, China; E-mail: hbcai@jsnu.edu.cn.
} 
Kijak \& Maron and Kijak et al. found some new GPS pulsars through the new observations, these observations indicated that the turn-over of pulsar's spectra is related to its surroundings [3-4]. Kijak et al. launched the multi-epoch multi-band observation campaign for the binary system B1259-63, and they found that the spectra of B1259-63 will vary with its distance from the companion star [5]. The spectra of B1259-63 will begin to turn over when it approaches the companion star, then the turnover spectra of B1259-63 vanishes when this pulsar is far away the companion star. These observations strongly prove the turn-over spectra of pulsar is caused by its surroundings, i.e., the free-free absorption is the main mechanism forming the turnover spectra. Kijak further probed into the origin of interstellar medium, which may be the supernova remnants, hydrogen ionization region, pular wind nebula [6].

According to the estimate from Bates et al. there may be about $10 \%$ GPS pulsars in the total pulsars, but the number of identified GPS pulsars is only 23 which is much lower than the estimate [7]. For searching more GPS pulsars, it is necessary to understand the statistical properties of these GPS pulsars. In this paper, we will study the relations among the parameters of GPS pulsars, such as the peak frequencies, spectral indexes, dispersion measures, and the period of these GPS pulsars.

\section{Radiation Mechanism of GPS Pulsars}

Kijak et al. studied the spectra of GPS pulsars, and they thought the turn-over spectra of GPS pulsar is caused by free-free absorption [5]. The electrons in the interstellar medium can emit photons by the free-free radiation, but also absorb the photons passing through the interstellar medium, the free-free absorption makes the photons transiting from the low free state to high free state. The absorbed photons may come from the internal free-free radiation, or come from the external radiation such as cosmic background radiation or the radio radiation from the active galactic nucleus (AGN). FFA is also used to explain the turn-over spectra of AGN [8]. According to the FFA model, we can know that the interstellar medium has low effect on the pulsar's radiation in the high frequency, and the spectra of pulsar show the power law form. In the low frequency band, the power-law spectra begins to turn over due to the free-free absorption. The spectral shape of GPS pulsar is as figure 1 .

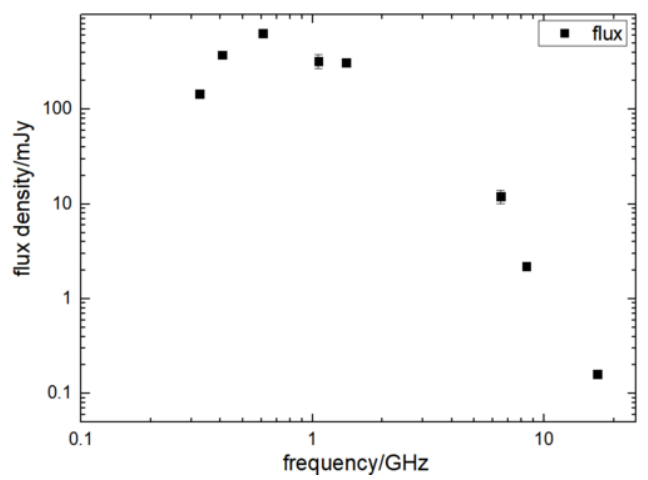

Figure 1. The spectra of PSR J1644-4559（B1641-45） 


\section{Statistical Analysis of GPS Pulsars}

\subsection{Peak frequency Statistics of GPS Pulsar}

We obtained 23 GPS pulsars through the literature research, the detailed pulsars and their parameters are listed in table 1 . The peak frequency of these pulsars are all above $0.5 \mathrm{GHz}$, figure 2 shows the statistical distribution of peak frequency. We can see from figure 2 that 17 pulsars' peak frequencies locate between $0.5-1 \mathrm{GHz}$, the ratio is $73.9 \%$. The average peak frequency of the 17 pulsars is $0.66 \mathrm{GHz}$. The number with the peak frequencies above $1 \mathrm{GHz}$ is six, among which there are four pulsars with the peak frequencies above $3 \mathrm{GHz}$, especially the peak frequency of $\mathrm{J} 1745-2900$ is $6.3 \mathrm{GHz}$ which is the highest peak frequency currently known [9]. J1745-2900 is a magnetic star locating in the center of Galaxy, and its peak frequency is not always invariant. The research from Lewandowski et al. shows J1745-2900 was observed to have a burst in the X-ray in April 2013, subsequently many radio telescopes over the world launched the monitoring for the pulsar [9]. Lewandowski et al. found that the peak frequency of $\mathrm{J} 1745-2900$ is $6.3 \mathrm{GHz}$ on May 1 st 2013 , then the peak frequency became as $3.5 \mathrm{GHz}$ [9]. Pennucci found that its peak frequency had moved to $2 \mathrm{GHz}-2.5 \mathrm{GHz}$ after 100 days since J1745-2900's bust in April 2013 [10]. Lewandowski et al. used the FFA model to study the variation of the magnetic star's spectra, and they thought the interstellar medium around the magnetic star will expand rapidly after the magnetic star's burst, and correspondingly the electron density in the interstellar medium will reduce due to the expanding of interstellar medium [9]. The reducing of electron density will result in FFA optical depth dwindling, and leads to the peak frequency moving to lower frequency.

The other two magnetic stars J1550-5418 and J1622-4950 also show high peak frequencies, which exceed 3GHz [11]. Three magnetic stars ( J1745-2900, J1745-2900, J1745-2900) all have high turn-over peak frequencies, which indicates that magnetic stars may be surrounded by high-density interstellar medium, which can be confirmed by the high dispersion measures of the three magnetic stars in table 1 . We can also find from table 1 that J1550-5418 and J1622-4950 have flatter spectra in the high frequency band. Although J1745-2900 has the steep spectral index, its spectra has the flatting trend after the burst in April 2013 [10, 12].

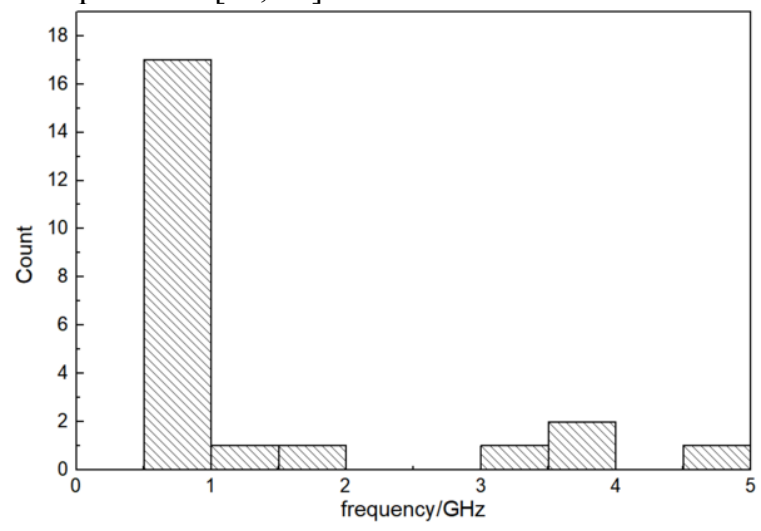

Figure 2. Distribution of peak-frequency of GPS pulsars 
The variation of peak frequency is also found in the pulsar binary system PSR B1259-63/LS2883. PSR B1259-63 makes the orbital motion around the companion LS2883, the peak frequency of PSR B1259-63 will also vary with the distance between PSR B1259-63 and LS2883.

Table 1. Gigahertz-peaked Pulsars and their parameters. Pulsar with asterisk is the magnetic star.

\begin{tabular}{|c|c|c|c|c|c|c|c|}
\hline Pulsar & $\operatorname{Dm}\left(\mathrm{pc} \mathrm{cm}^{-3}\right)$ & $\operatorname{age}(\mathrm{kyr})$ & $\begin{array}{l}\text { Spectral } \\
\text { index }\end{array}$ & $\begin{array}{l}\text { Peak } \\
\text { frequency/ } \\
\text { GHz } \\
\end{array}$ & $\mathbf{P} / \mathbf{s}$ & P1 & References \\
\hline B0833-45 & 67.97 & 11.3 & -2.9 & 0.56 & 0.089 & $1.25 \mathrm{E}-13$ & {$[13]$} \\
\hline B1054-62 & 320 & 1870 & -1.33 & 0.59 & 0.42245 & $3.58 \mathrm{E}-15$ & {$[11]$} \\
\hline B1259-63 & 147 & 330 & -1.3 & 3.6 & 0.04776 & $2.28 \mathrm{E}-15$ & [14] \\
\hline B1641-45 & 479 & 359 & -2.79 & 0.58 & 0.45508 & 2.01E-14 & {$[11]$} \\
\hline B1800-21 & 238 & 15.4 & -1.06 & 0.76 & 0.13367 & $1.34 \mathrm{E}-13$ & [11] \\
\hline B1822-14 & 357 & 195 & -0.65 & 0.6 & 0.2792 & $2.27 \mathrm{E}-14$ & [11] \\
\hline B1823-13 & 231 & 21.4 & -0.68 & 0.9 & 0.10149 & 7.53E-14 & [11] \\
\hline B1828-11 & 161 & 107 & -2.62 & 0.83 & 0.40507 & $5.99 \mathrm{E}-14$ & {$[11]$} \\
\hline J1550-5418* & 830 & 1.41 & -0.46 & 3.23 & 2.06983 & $2.32 \mathrm{E}-11$ & [11] \\
\hline J1622-4950* & 820 & 4.03 & -0.54 & 3.73 & 4.32702 & $2.78 \mathrm{E}-12$ & {$[11]$} \\
\hline $\mathrm{J} 1702-4128$ & 367.1 & 5.51 & -0.55 & 0.55 & 0.18214 & $5.23 \mathrm{E}-14$ & [15] \\
\hline J1718-3825 & 247.4 & 8.95 & -0.82 & 1.03 & 0.07467 & $1.32 \mathrm{E}-14$ & {$[15]$} \\
\hline J1723-3659 & 254 & 401 & -1.8 & 0.56 & 0.20272 & 8.03E-15 & {$[11]$} \\
\hline $\mathrm{J} 1740+1000$ & 24 & 144 & -0.75 & 0.54 & 0.15409 & $2.15 \mathrm{E}-14$ & {$[16]$} \\
\hline J1745-2900* & 1778 & 3.4 & -1.5 & 4,5 & 3.76373 & $1.76 \mathrm{E}-11$ & {$[9]$} \\
\hline $\mathrm{J} 1747-2958$ & 101.5 & 2.55 & -0.85 & 0.73 & 0.09881 & $6.13 \mathrm{E}-14$ & {$[15]$} \\
\hline J1809-1917* & 197.1 & 5.13 & -1.0 & 1.93 & 0.08276 & $2.55 \mathrm{E}-14$ & {$[11]$} \\
\hline J1835-1020 & 114 & 810 & -1.8 & 0.55 & 0.30245 & $5.92 \mathrm{E}-15$ & [11] \\
\hline J1841-0345 & 194 & 55.9 & -0.78 & 0.55 & 0.20407 & $5.79 \mathrm{E}-14$ & {$[11]$} \\
\hline J1852-0635 & 171 & 567 & -0.69 & 0.9 & 0.52416 & $1.46 \mathrm{E}-14$ & {$[11]$} \\
\hline $\mathrm{J} 1901+0510$ & 429 & 313 & -1.8 & 0.52 & 0.61476 & $3.11 \mathrm{E}-14$ & [11] \\
\hline $\mathrm{J} 1907+0918$ & 358 & 38 & -2.8 & 0.59 & 0.22611 & $9.43 \mathrm{E}-14$ & {$[11]$} \\
\hline $\mathrm{J} 2007+2722$ & 127 & 404000 & -0.85 & 0.79 & 0.0245 & $9.61 \mathrm{E}-19$ & {$[11]$} \\
\hline
\end{tabular}

\subsection{Distribution of Spectral Index}

The statistical distribution of spectral index of GPS pulsars is shown in figure 3, from which we can see that there 11 pulsars with the spectral index above -1.0 , the proportion is $47.8 \%$ in the total sample. Malofeev carried out a statistics on 284 pulsars and obtained the average spectral index was -1.71. Maron et al. gave the average spectral index of 281 pulsars was -1.8 [1]. According to our statistics, the average spectral index of these GPS pulsars is -1.33 which is higher than the ordinary pulsars. 


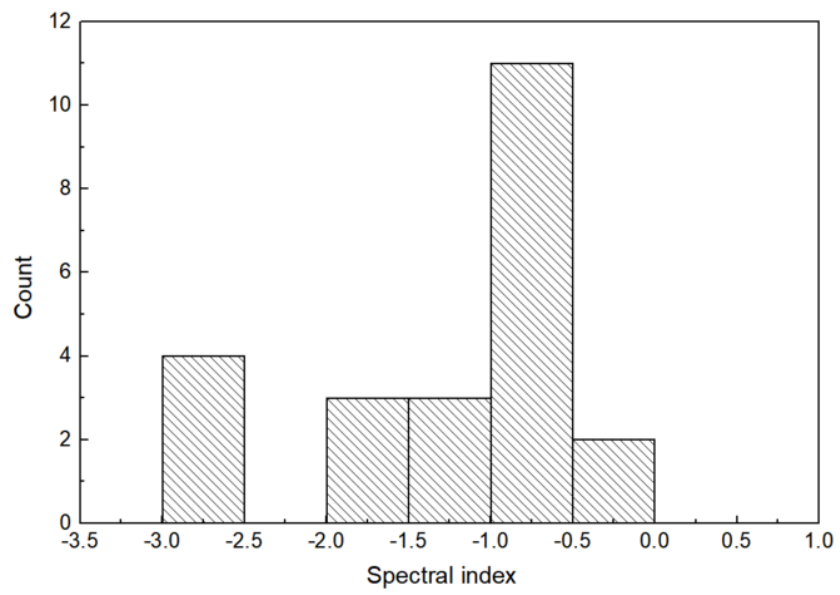

Figure 3. Distribution of spectral indexes of GPS pulsars

From table 1, we can find that the average spectral indexes of four magnetic stars is -0.88 , higher than other GPS pulsars, which indicates their spectra in the high frequency band will be flatter than other pulsars. The conclusion is consistent with the others' research [17]. J1809-1917 is the first detected magnetic star in the radio band, Basu et al. obtained its spectral index as -1.0, but Dai et al. (2019) gave its new spectral index as 0.3 [15]. We found the observations in Dai et al. (2019) was during the radio burst stage of J1809-1917, and the observations in Basu et al. (2018) was during the radio quiet stage of J1809-1917. From the spectra given by Dai et al., we can also find that J1809-1917 has higher peak frequency during its radio burst stage [18].

Kijak et al. used the published data to fit the spectra of J1550-5418, and obtained its spectral index as -0.46 . In further [11], Chu et al. gave its spectral index as 0.4 in the $43 \mathrm{GHz}-100 \mathrm{GHz}$ band, which indicates that there may be a peak about the spectra of J1550-5418 in the higher frequency [19]. The high frequency character of J1745-2900 is similar to the one of J1550-5418, the spectra of J1745-2900 may have a peak near $200 \mathrm{GHz}$. It is difficult to explain the higher frequency peak of the GPS pulsars by using the FFA model because the optical depth is small in the higher frequency band. The peak of spectra in $100 \mathrm{GHz}-200 \mathrm{GHz}$ may be due to incoherent radiation according to [20]. Michel thought the radiation in the lower frequency band than $100 \mathrm{GHz}$ is coherent radiation, and yet the incoherent radiation begins to enhance in the higher frequency band than $100 \mathrm{GHz}[20]$.

We also find four GPS pulsars having very steep spectral index, the average spectral index of these pulsars is -2.78 which is much lower than ordinary pulsars' spectral index, -1.8 , given by [1].

\subsection{Distribution of Dispersion Measure}

The radiation of pulsars will be scattered by the free electrons when it transit the interstellar medium, which results in the different delay when we receive the radiation in the differenct frequency, i.e., the dispersion measure (DM) phenomenon. According to Cordes, DM is proportion to the distance of pulsar from us, also proportional to the electron density along the propagation path [21]. According to Kijak et al., the cause 
forming the turn-over spectra may be FFA by the electrons in the interstellar medium, so the electron density along the propagation path of GPS pulsar radiation will be high, in further DMs of GPS pulsars will also be high $[5,11]$. We give the statistic DM distributon of GPS pulsars in figure 4 . We can see from figure 4 that 9 pulsars have DM less than 200pc cm${ }^{-3}$, the ones of others' DM are all greater than $200 \mathrm{pc} \mathrm{cm}-3$ and the average DM is $541 \mathrm{pc} \mathrm{cm}^{-3}$. The magnetic star J1745-2900 has the greatest DM in the sample which proves that FFA is the important factor causing the turn-over spectra of pulsars.

\subsection{Correlation Between Pulsar's Period and DM}

We gave the correlation between the periods and DMs of GPS pulsars in figure 5, the Pearson correlation coefficient is 0.85 . We can see from figure 5 the strong positive correlation between the periods and DMs of GPS pulsars, DM will increase with the increase of period. The data point of J1622-4950 has big divergence from the fitting cure, it may result from the underestimate of DM of J1622-4950. J1622-4950 is a young magnetic star which is in the active phase, so the density of interstellar medium may also vary violently. This variation will result the fluctuation of DM. Scholz et al. gave the flux density variation of J1622-4950 with time, which is in the drastic changes [22]. The drastic change of flux density may be due to the variation of surroundings of $\mathrm{J} 1622-4950$. So it is necessary to remeasure DM of J1622-4950 for verifying the correlation between the periods and DMs of GPS pulsars.

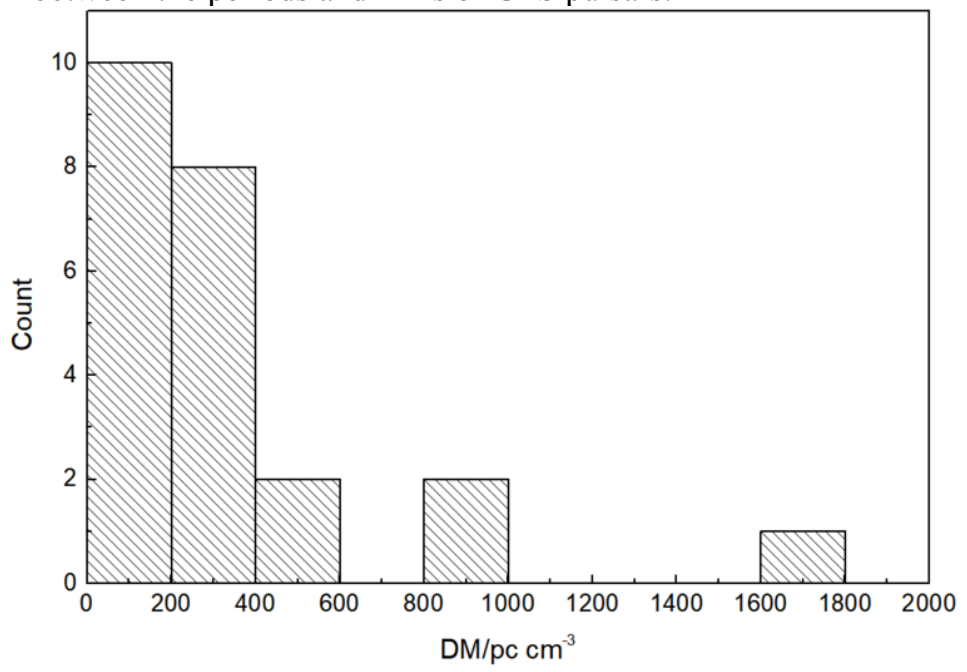

Figure 4. Distribution of DM of GPS pulsars 


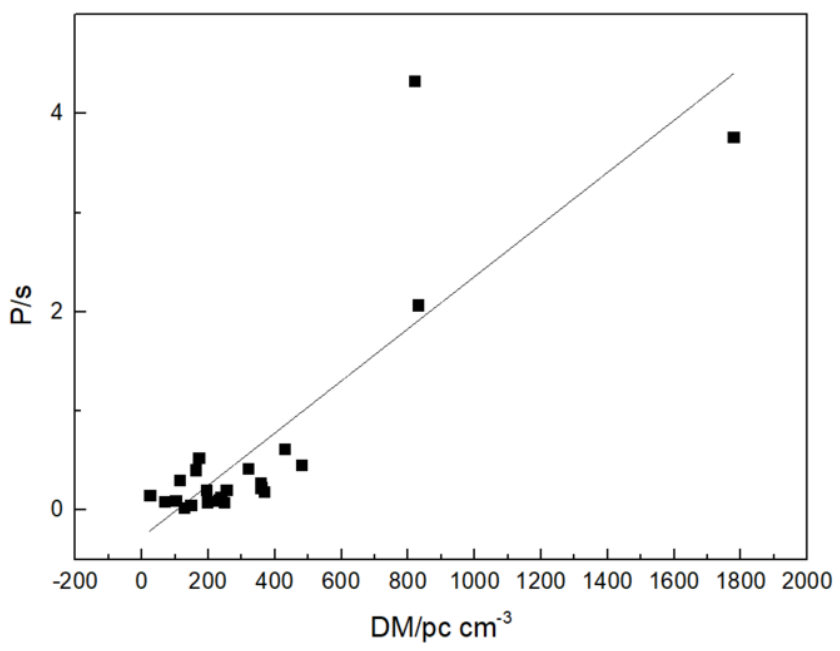

Figure 5. Correlation between DM and the period of pulsar

\section{Discussion and Conclusion}

We have a statistical research on 23 GPS pulsars, and find the peak frequency of most GPS pulsars is between $0.5 \mathrm{GHz}-1.0 \mathrm{GHz}$, the proportion is $73.9 \%$. The magnetic star have higher peak frequency in general than other GPS pulsars, the average peak frequency of 4 magnetic stars is $3.35 \mathrm{GHz}$. The mean spectral index of GPS pulsars is 1.33 which is higher than the one of previous statistics on ordinary pulsars, -1.80 . The flattening of GPS pulsars may be due to that the interstellar medium has obvious suppress on the low-frequency radiation of GPS pulsars. GPS pulsar has high DM, the average DM of these GPS pulsars is $348 \mathrm{pc} \mathrm{cm}^{-3}$. We also find the strong positive correlation between the periods and DMs of these GPS pulsars, the larger the dispersion, the larger the period of pulsar. This correlation may indicate that the friction drag on the GPS pulsars from the interstellar medium may be the important reason for the speed reduction of pulsars. It deserves the further study whether or not this correlation exist in other kind of pulsars.

\section{Acknowledgments}

This work was supported by Key Research \& Development projects in Xuzhou (Grant No. KC 18079).

\section{References}

[1] Maron O., Kijak J., Kramer M., Wielebinski R., 2000, A\&AS, 147, 195

[2] Koribalski B., Johnston S., Weisberg J.M., Wilson W., 1995, ApJ, 441, 756

[3] Kijak J., Maron O., 2004, IAU Symposium, Vol. 218, 2004

[4] Kijak J., Gupta Y., Krzeszowski K., 2007, A\&A, 462, 699

[5] Kijak J., Lewandowski W., Maron O., Gupta Y., Jessner A., 2011, A\&A, 531, A16

[6] Kijak J., Lewandowski W., Rozko K., 2018, IAUS, 337, 352 
[7] Bates S. D., Lorimer D. R., Verbiest J. P. W., 2013, MNRAS, 431, 1352

[8] Kameno S., Horiuchi S., Shen Z.-Q., Inoue M., KobayaDai H., HirabayaDai H., Murata Y., 2000, PASJ, 52,209

[9] Lewandowski W., Rozko K., Kijak J., Melikidze G.I., 2015, ApJ, 808, 18

[10] Pennucci T.T., Possenti A., Esposito P., Rea N., Haggard D., Baganoff F. K., Burgay M., et al., 2015, ApJ, 808, 81

[11] Kijak J., Basu R., Lewandowski W., Ro\{\.z\}ko K., Dembska M., 2017, ApJ, 840, 108

[12] Torne P., Eatough R.P., Karuppusamy R., Kramer M., Paubert G., Klein B., Desvignes G., et al., 2015, MNRAS, 451, L50

[13] Sieber W.,1973, A\&A, 28, 237

[14] Dembska M., Basu R., Kijak J., Lewandowski W., 2015, MNRAS, 449, 1869

[15] Basu R., Rozko K., Kijak J., Lewandowski W., 2018, MNRAS, 475, 1469

[16] Rozko K., Kijak J., Chyzy K., Lewandowski W., Daimwell T., Sridhar S.S., Curylo M., et al., 2020, ApJ, 903, 144

[17] Keith M.J., Johnston S., Levin L., Bailes M., 2011, MNRAS, 416, 346

[18] Dai S., Lower M. E., Bailes M., Camilo F., Halpern J. P., Johnston S., Kerr M., et al., 2019, ApJL, $874, \mathrm{~L} 14$

[19] Chu C.-Y., Ng C.-Y., Kong A.K.H., Chang H.-K., 2021, MNRAS, 503, 1214

[20] Michel F.C., 1982, PhRvL, 48, 580

[21] Cordes J.M., 1978, ApJ, 222, 1006

[22] Scholz P., Camilo F., Sarkissian J., Reynolds J. E., Levin L., Bailes M., Burgay M., et al., 2017, ApJ, 841,126 